>ADAMTS13 (A disintegrin and metalloprotease with thrombospondin type 1 repeats) est la protéase spécifique du facteur von Willebrand (VWF). $\varepsilon$ n cas de brèche vasculaire, le VWF permet, grâce à sa structure multimérique, l'adhésion des plaquettes au sous-endothélium et l'agrégation des plaquettes entre elles dans la microcirculation, où les forces de cisaillement sont élevées. ADAMTS13 régule l'activité du VWF en réduisant la taille de ses multimères. Un déficit fonctionnel sévère en ADAMTS13 est observé dans la majorité des cas de purpura thrombotique thrombocytopénique (PTT), une microangiopathie thrombotique définie par la formation spontanée, dans la microcirculation sanguine, de thrombus plaquettaires responsables d'une anémie hémolytique mécanique, d'une thrombopénie de consommation et de signes d'ischémie multiviscérale. II s'agit d'une maladie rare ( 4 cas $/ 10^{6}$ habitants/an) mais gravissime en l'absence de traitement immédiat et spécifique (plasmathérapie). Dans $90 \%$ des cas, le PTT est acquis et dû à la présence d'autoanticorps anti-ADAMTS13. Dans les autres cas, il s'agit d'un déficit constitutionnel, de transmission autosomique récessive, appelé syndrome d'Upshaw-Schulman. Une meilleure caractérisation structurale et fonctionnelle d'ADAMTS13 combinée aux études cliniques menées chez les patients atteints de PTT est cruciale pour évaluer la pertinence d'une forme purifiée plasmatique ou recombinante à visée thérapeutique. <

L'hémostase correspond aux différents mécanismes mis en jeu pour réparer une brèche vasculaire. Une première étape, l'hémostase primaire, correspond à la formation du clou plaquettaire puis une seconde étape, la coagulation, aboutit à la formation du caillot de fibrine. Le facteur von Willebrand (VWF) est une glycoprotéine plasmatique qui joue un rôle clé dans l'hémostase

\section{ADAMTS13, \\ la protéase \\ spécifique \\ du clivage du facteur von Willebrand}

Agnès Veyradier, Paul Coppo

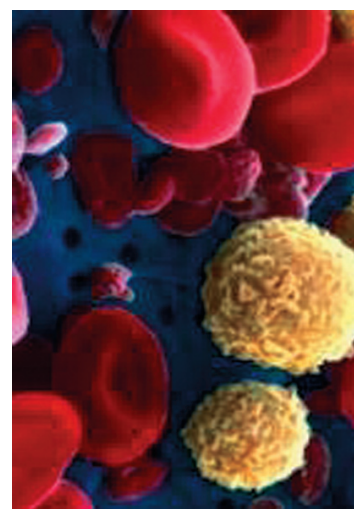

A. Veyradier : Service

d'hématologie biologique, hôpital Antoine Béclère, groupe hospitalier Paris-Sud, AP-HP, 157, rue de la Porte de Trivaux, 92140 Clamart, France ; Inserm U770, Université Paris-Sud, 80, rue du Général Leclerc, 94276 Le Kremlin-Bicêtre ; Centre national de référence des microangiopathies thrombotiques de l'adulte et de l'enfant (CNR-MAT), Paris, France.

primaire puisqu'il est indispensable à l'adhésion des plaquettes au sous-endothélium mis à nu par la brèche vasculaire et à l'agrégation des plaquettes entre elles à taux de cisaillement élevés du flux sanguin (représentatifs de la microcirculation sanguine) [1]. Le VWF est synthétisé dans les cellules endothéliales et dans les mégacaryocytes et stocké dans des compartiments cellulaires à P. Coppo: Département d'hématologie clinique, hôpital Saint-Antoine, groupe hospitalier Paris-Est, AP-HP, 184, rue du Faubourg Saint-Antoine, 75012 Paris ; Centre national de référence des microangiopathies thrombotiques de l'adulte et de l'enfant (CNR-MAT), Paris, France.

agnes.veyradier@abc.aphp.fr partir desquels il est secrété dans le plasma. La particularité du VWF est d'avoir une structure multimérique organisée en association de dimères (eux-mêmes constitués de l'association de deux monomères dont la masse moléculaire est de l'ordre de $250 \mathrm{kDa}$ ) : le plus petit multimère est un dimère de $500 \mathrm{kDa}$ et les plus grands multimères peuvent atteindre 30000 kDa. Cette organisation multimérique a une conséquence directe sur la fonction du VWF puisque son pouvoir adhésif vis-à-vis du sous-endothélium et des plaquettes est proportionnel à la taille du multimère. Dans le plasma circule un éventail de multimères du VWF de différentes tailles, classés en 3 groupes: les bas PM, les PM intermédiaires et les hauts PM (HPM). Dans les compartiments cellulaires, il existe en plus des multimères de très hauts PM (THPM) du VWF qui ne sont pas retrouvés dans le plasma dans les conditions physiologiques. La régulation de la taille des multimères de VWF dans le plasma repose essentiellement sur l'action d'une protéase, ADAMTS13 (A disintegrin and metalloprotease with thrombospondin type 1 repeats, $13^{\mathrm{e}}$ membre), spécifique du clivage 
du VWF [1]. Cette régulation est cruciale pour prévenir la formation de thrombus plaquettaires au sein de la microcirculation sanguine.

\section{ADAMTS13 : biosynthèse, structure, propriétés biochimiques, fonction}

\section{Gène et précurseur protéique d'ADAMTS13}

Le gène codant pour ADAMTS13 a été identifié grâce simultanément au séquençage de l'extrémité amino-terminale de la protéine [2-4] et à une stratégie de clonage positionnel [5] conduisant ainsi à la découverte du $13^{e}$ membre de la famille ADAMTS dont une vingtaine de membres sont identifiés (Figure 1). Le gène codant pour ADAMTS13 (ADAMTS13) est localisé sur le chromosome 9q34 et s'étend sur 37 kb incluant 29 exons. L'ARNm complet comprend 4,6 kb, mais il existe un épissage alternatif du gène conduisant à plusieurs variants dont la signification demeure inconnue. ADAMTS13 est synthétisée principalement par les cellules stellaires périsinusoïdales (ou cellules de Ito siégeant entre les hépatocytes et les cellules endothéliales) du foie [6] mais aussi par les cellules endothéliales [7] et les cellules de la lignée mégacaryocytaire [8]. Elle est secrétée dans le plasma sous forme d'une enzyme active d'environ $200 \mathrm{kDa}$.
La pré-pro-ADAMTS13 est une monochaîne de 1427 acides aminés (aa), schématisée dans la Figure 2, qui comprend un domaine amino-terminal caractéristique des protéines de la famille et une combinaison spécifique carboxy-terminale [3].

\section{Importance des conditions hémodynamiques} pour l'activité d'ADAMTS13

Les principales propriétés biochimiques d'ADAMTS13 ont pu être déterminées dès 1996 grâce à sa purification partielle à partir de plasma humain $[9,10]$. ADAMTS13 est différente des métalloprotéases matricielles ou des sérine-protéases plasmatiques puisque les inhibiteurs habituels de celles-ci n'ont aucun effet sur sa propre activité enzymatique in vitro. Son spectre d'action apparaît étroit puisqu'elle ne dégrade ni le fibrinogène, ni l'albumine, ni le collagène. $\varepsilon$ n revanche, elle hydrolyse spécifiquement le VWF (dans un domaine dit A2) par scission du pont peptidique Tyr1605-Met1606. In vitro, son activité enzymatique est potentialisée par les cations divalents (en particulier le $\mathrm{Ba}^{2+}$ ) et par un $\mathrm{pH}$ optimum compris entre 8 et 9 ; elle est bloquée en présence d'EDTA. Sa concentration plasmatique est de l'ordre de $1 \mu \mathrm{g}$ / $\mathrm{ml}$. Sa demi-vie dans le plasma, comprise entre 2 et 3 jours, est inhabituellement longue pour une protéase [11] ce qui suggère l'existence, en plus de sa forme soluble plasmatique, d'une forme liée à un récepteur cellulaire et/ou à un transporteur plasmatique qui n'est pas encore identifié. La seule fonction d'ADAMTS13 connue à ce jour est de cliver spécifiquement les multimères plasmatiques du VWF afin d'en limiter la taille et ainsi le pouvoir adhésif vis-à-vis des plaquettes.

La régulation de l'activité d'ADAMTS13 sur le VWF fait intervenir des propriétés intrinsèques au VWF qui dépendent très directement des conditions hémodynamiques. En effet, la modélisation du domaine A2 du VWF suggère que le pont peptidique Tyr 1605-Met1606, qui constitue le site spécifique de clivage par ADAMTS13, est cryptique au sein 


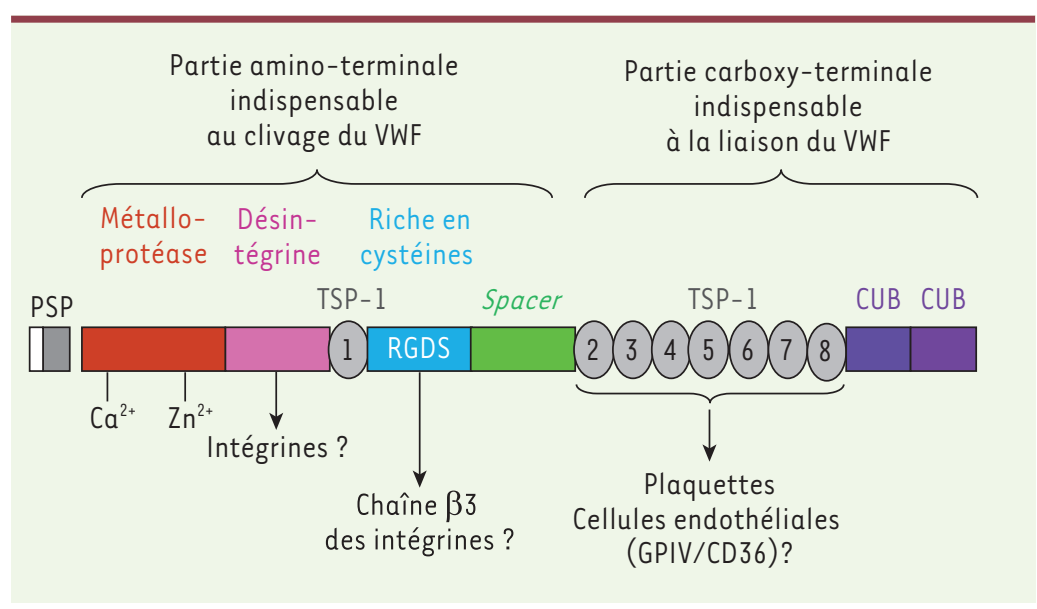

Figure 2. Domaines structuraux et fonctionnels d'ADAMTS13. ADAMTS13 est une glycoprotéine monocaténaire composée de la succession des domaines structuraux suivants : un peptide signal (PS, $33 \mathrm{aa}$ ), un propeptide $(P, 4 l \mathrm{aa})$ dont la séquence carboxyterminale est compatible avec une protéolyse par la furine. Après clivage du propeptide, la forme mature d'ADAMTS13 comprend : un domaine métalloprotéase incluant le site actif enzymatique $H^{224} \varepsilon X X$ HXXGXXHD ${ }^{235}$ de type adamalysine qui contient des sites de liaison aux ions $\mathrm{Ca}^{2+}$ et $\mathrm{Zn}^{2+}$; un domaine de type désintégrine; un domaine TSPl ; un domaine riche en cystéines contenant une séquence RGDS potentiellement impliquée dans des interactions avec la chaîne $\beta 3$ des intégrines; un domaine de type ADAMTS spacer ; sept autres domaines TSPl indispensables à son interaction avec les plaquettes et les cellules endothéliales et deux domaines CUB. La partie amino-terminale d'ADAMTS13 (allant du domaine métalloprotéase jusqu'au domaine spacer inclus) est indispensable au clivage du facteur Willebrand in vitro et in vivo. La partie carboxy-terminale d'ADAMTS13 n'est pas indispensable à ce clivage in vitro mais elle l'est probablement in vivo, puisqu'elle est indispensable à la liaison d'ADAMTS13 au facteur Willebrand d'une part et à son récepteur candidat sur les cellules endothéliales, le CD36, d'autre part. La protéase mature est glycosylée et cela explique probablement la différence entre sa masse moléculaire calculée $(145 \mathrm{kDa})$ et la masse moléculaire apparente de son produit de purification plasmatique $(190 \mathrm{kDa})$

de la molécule de VWF native [12]. Ainsi, seul le dépliement du VWF induit soit par les forces de cisaillement élevées du flux sanguin dans les microvaisseaux in vivo, soit par des agents dénaturants (urée, hydrochlorure de guanidine), ou son immobilisation sur une surface plastique [13] in vitro, permet l'exposition de ce pont peptidique et le rend donc accessible au clivage par ADAMTS13. De plus, cet accès est facilité par la liaison du VWF à certains ligands comme la P-sélectine (qui intervient dans la fixation du VWF à la surface des cellules endothéliales à la phase initiale de sa sécrétion dans le plasma [14]) ou la glycoprotéine Ib plaquettaire (qui permet la liaison du VWF aux plaquettes au sein du clou plaquettaire formé pour réparer une brèche vasculaire [15]). Ceci explique pourquoi le rôle d'ADAMTS13 dans la régulation de la taille des multimères du VWF est prédominant dès l'étape de sécrétion du VWF par les cellules endothéliales (limitation immédiate de la taille des multimères avant la mise en circulation du VWF dans le plasma) et aussi au sein du clou plaquettaire (résorption du clou plaquettaire une fois la brèche vasculaire réparée) (Figure 3) $[14,15]$.

\section{Domaines protéiques d'ADAMTS13 et interactions avec le VWF} Les sites précis de liaison entre le VWF et ADAMTS13 restent à identifier, tant sur le substrat que sur l'enzyme, de même que la séquence précise des interactions lors du processus de reconnaissance du substrat par l'enzyme. Des indications sont néanmoins disponibles grâce à une approche reposant essentiellement sur des expériences de liaison de protéines tronquées in vitro soit en conditions statiques, soit en conditions de flux (Figure 2). Le clivage du propeptide d'ADAMTS13 n'apparaît pas indispensable à son activité enzymatique [16]. Son domaine catalytique seul (métalloprotéase) ne suffit pas à assurer son activité enzymatique vis-à-vis du VWF, activité qui requiert la présence additionnelle du domaine riche en cystéines et du domaine spacer [17]. En revanche, les domaines thrombospondine de type l (TSPI) 2-8 et les domaines CUB $^{1}$ ne sont pas indispensables au clivage du VWF in vitro mais ils joueraient un rôle essentiel et coopératif dans le phénomène de reconnaissance du substrat [18]. En effet, ils pourraient permettre à ADAMTS13 de se fixer in vivo aux cellules endothéliales (le CD36 endothélial est un récepteur candidat [19]) ou aux plaquettes par son extrémité carboxy-terminale, cet ancrage cellulaire pouvant alors être un prérequis pour son interaction avec le VWF (Figure 3). L'interaction directe entre ADAMTS13 et le VWF impliquerait séquentiellement une liaison entre la séquence allant du TSP1 5 au CUB2 d'ADAMTS13 et les domaines D4-CK du VWF [20], une liaison entre le domaine CUB1 d'ADAMTS13 et le domaine A3 du VWF [21] puis une liaison entre les domaines riche en cystéines et spacer d'ADAMTS13 et le domaine A2 du VWF [22].

\section{Exploration biologique d'ADAMTS13}

En 2011, l'exploration biologique d'ADAMTS13 repose sur des tests phénotypiques plasmatiques (ou sériques) limités à la mesure de son activité protéolytique vis-à-vis du VWF et à celle de son antigène, ainsi qu'à son étude génétique par séquençage direct de son gène.

${ }^{1}$ Domaine CUB : acronyme formé des initiales de trois protéines possédant ces domaines : complement $(\mathrm{Clr} / \mathrm{Cls})$, sea urchin $E G F$, bone morphogenetic protein. 


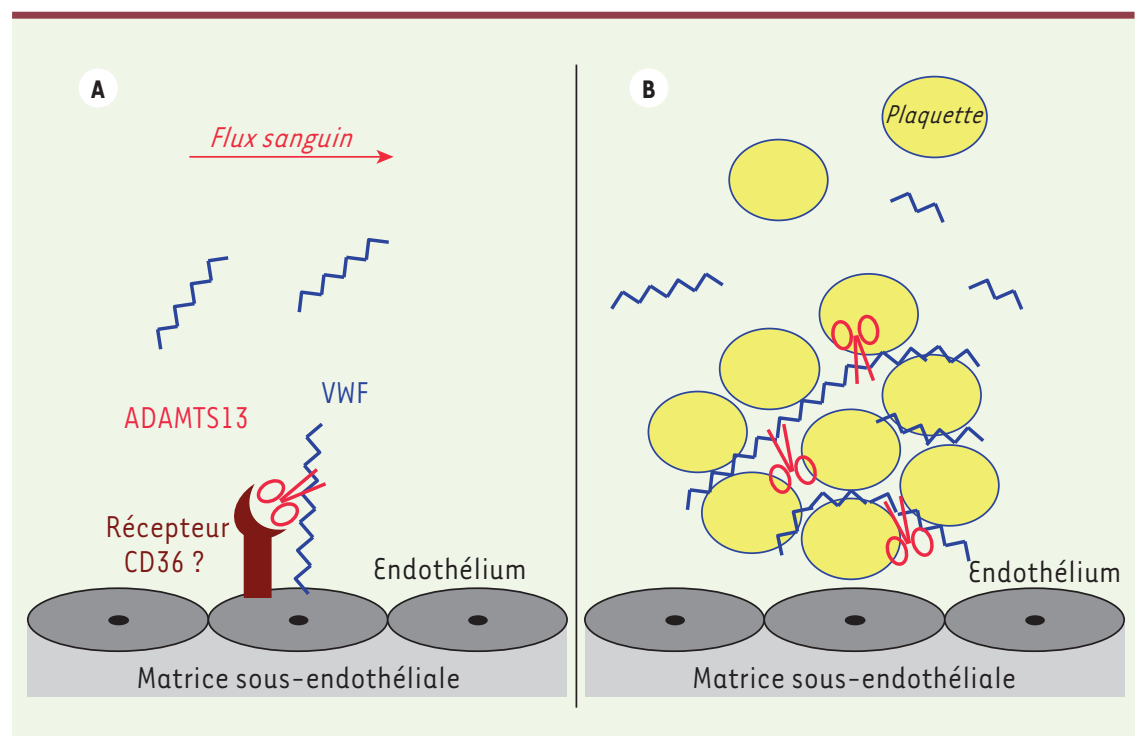

Figure 3. Clivage protéolytique du facteur Willebrand par ADAMTS13. ADAMTS13 intervient dans la régulation de la taille des multimères du facteur Willebrand (VWF) afin de limiter son pouvoir adhésif, dans deux situations distinctes. $A$. Premièrement, à la phase initiale de la sécrétion du VWF par les cellules endothéliales, ADAMTS13 clive les multimères de très haut poids moléculaire (THPM) du VWF (limitation immédiate de la taille des multimères du VWF avant sa mise en circulation dans le plasma). B. Deuxièmement, au sein du clou plaquettaire formé pour réparer une brèche vasculaire, ADAMTS13 clive les multimères de HPM du VWF liés aux plaquettes (résorption du clou plaquettaire une fois la brèche vasculaire réparée).

Depuis 1998, plusieurs méthodes de mesure de l'activité d'ADAMTS13 ont été développées. Toutes ces méthodes relèvent du statut d'examens hyperspécialisés récemment transférés de la recherche et non du statut d'examens spécialisés pouvant être validés en routine. En corollaire, ces examens, qui restent extrêmement coûteux et dont l'interprétation est souvent complexe, sont centralisés dans des laboratoires experts. Les méthodes de mesure de l'activité d'ADAMTS13 sont basées sur la dégradation d'un substrat exogène par l'ADAMTS13 du plasma testé $[23,24]$. Ce substrat est soit du VWF natif (molécule entière, VWF full-length) purifié à partir de plasma humain ou recombinant, soit de courts peptides synthétiques de VWF (substrat minimal pour ADAMTS13 composé d'une séquence de quelques aa incluant le site de clivage par ADAMTS13). Les produits de dégradation du VWF sont mesurés soit par électrophorèse, soit par des techniques immunologiques, soit par des techniques d'agrégation plaquettaire [23, 24]. Quelle que soit la méthode utilisée, les normes de l'activité d'ADAMTS13 se situent entre 50 et 150 \% (exprimées par rapport à un mélange de plasmas normaux).

Les sensibilité, spécificité, linéarité et précision de ces différentes méthodes ont été comparées dans différentes études multicentriques $[25,26]$. De manière synthétique, il apparaît que toutes les méthodes de mesure de l'activité d'ADAMTS13 ont le plus souvent un seuil de détection situé entre $5 \%$ et $10 \%$, et que leur sensibilité et leur spécificité sont excellentes dans la zone de pertinence clinique (déficit fonctionnel sévère d'ADAMTS13 défini par une activité inférieure à $10 \%$ ) pour le diagnostic positif de purpura thrombotique thrombocytopénique (PTT) (voir ci-après). Dans la zone des déficits partiels en ADAMTS13 (entre $15 \%$ et $50 \%$ ) dont la pertinence clinique n'est pas établie, il existe une assez grande variabilité inter-méthodes alors que, pour des valeurs normales supérieures à $50 \%$, les méthodes sont assez bien corrélées. Un certain nombre de coffrets commerciaux sont disponibles depuis quelques années sur le marché, mais leur performance n'a pas encore été évaluée par rapport aux techniques de référence dans le cadre d'études multicentriques.

Plusieurs tests (ELISA utilisant des anticorps [Ac] antiADAMTS13 polyclononaux ou monoclonaux, avec détection chromogénique ou Western blot) dédiés à la mesure de l'antigène ADAMTS13 sont disponibles. Les normes des taux d'antigène ADAMTS13 plasmatique sont comprises entre 700 et $1400 \mathrm{ng} / \mathrm{ml}$. En raison des différents mécanismes pouvant conduire à un déficit fonctionnel sévère d'ADAMTS13 (voir ci-après), la valeur du taux d'antigène ADAMTS13 n'est le plus souvent pas corrélée à l'activité d'ADAMTS13; par conséquent, c'est un paramètre de faible intérêt dans l'exploration biologique du PTT.

L'étude génétique d'ADAMTS13 repose sur un séquençage exhaustif des 29 exons et des jonctions exon-intron du gène ADAMTS13 puisqu'il n'existe pas de sites préférentiels de mutations dans le PTT héréditaire (syndrome d'Upshaw-Schulman), seule pathologie liée à un défaut génétique d'ADAMTS13 (voir plus loin).

\section{Variations physiologiques et pathologiques de l'activité d'ADAMTS13}

Les conditions physiologiques qui semblent influencer ADAMTS13 sont les âges extrêmes de la vie et la grossesse $[1,27]$. Ces conditions ont en commun une augmentation physiologique des taux de VWF et il apparaît donc que l'activité circulante d'ADAMTS13 varie en sens inverse du taux de VWF plasmatique [27]. Chez les nouveau-nés et les sujets âgés, l'activité d'ADAMTS13 apparaît discrètement diminuée [27, 28] d'environ 10 à 20 \% par rapport à la population générale. Au cours de 
la grossesse, l'activité d'ADAMTS13 diminue progressivement à partir de la $12^{\mathrm{e}}$ semaine d'aménorrhée pour atteindre, à terme, des taux $15 \%$ à $20 \%$ inférieurs au taux de base [29]. Des variations ethniques ont également été décrites : les sujets noirs ont des taux d'ADAMTS13 plus bas et cela serait dû à leur taux de VWF physiologiquement plus élevé [1].

\section{Déficits fonctionnels partiels en ADAMTS13}

Les déficits fonctionnels partiels en ADAMTS13 sont définis par des activités comprises entre 20 et $50 \%$. Contrairement au déficit sévère qui est pathognomonique du PTT (voir ci-après), ils ne sont pas spécifiques puisque retrouvés dans de nombreuses pathologies: pathologies cardiovasculaires, sepsis sévère, accès palustre, syndrome des antiphospholipides, certaines microangiopathies thrombotiques (MAT) comme le HELLP syndrome (hemolysis elevated liver enzymes low platelet count) ou le syndrome hémolytique et urémique [30]. Dans environ $10 \%$ des cas, des tableaux cliniques de PTT peuvent également s'accompagner d'une activité d'ADAMTS13 subnormale ; le plus souvent, ces PTT surviennent dans un contexte de cancer, de pathologie liée au VIH (virus de l'immunodéficience humaine) ou de greffe [30]. Quelle que soit la pathologie concernée, la pertinence clinique de ces déficits partiels en ADAMTS13 n'est pas clarifiée. Les mécanismes de ces déficits fonctionnels partiels en ADAMTS13 sont multiples: diminution de la synthèse et/ou de la sécrétion d'ADAMTS13 liée à une dysfonction de l'endothélium, consommation de l'enzyme par un excès de substrat, inhibition d'ADAMTS13 par certaines cytokines proinflammatoires comme l'IL6, dégradation d'ADAMTS13 par des enzymes protéolytiques (thrombine, élastases leucocytaires, plasmine) [30].

\section{Déficit fonctionnel sévère en ADAMTS13 :}

\section{le purpura thrombotique thrombocytopénique}

Le déficit fonctionnel sévère en ADAMTS13 (activité < $10 \%$ ) est pathognomonique d'une forme particulière de microangiopathie thrombotique appelée PTT $[1,30]$. Le PTT est défini par la formation spontanée dans la microcirculation sanguine de thrombus plaquettaires responsables d'une anémie hémolytique mécanique (cassure des globules rouges sur les thrombus intravasculaires produisant des schizocytes), d'une thrombopénie de consommation et de signes d'ischémie multiviscérale touchant principalement le cerveau et le rein. II s'agit d'une maladie rare dont l'incidence est estimée à quatre cas pour un million d'habitants et par an. À l'exception des rarissimes formes à révélation pédiatrique, le PTT touche principalement l'adulte jeune de 30 à 40 ans avec une prédominance féminine $(2 \mathrm{~F} / \mathrm{lH})$. Le PTT évolue classiquement par poussées entrecoupées de périodes de rémission clinique. L'intensité du tableau clinique lors d'une poussée est très hétérogène allant de formes frustes (thrombopénie isolée) jusqu'à des formes gravissimes de défaillance multiviscérale ischémique; de même, la fréquence des poussées est très variable (sporadiques ou au contraire récurrentes jusqu'à la chronicité) et reste imprévisible. Dans plus de $50 \%$ des cas, le PTT est idiopathique; dans les autres cas, il survient dans un contexte clinique plus large, associé à d'autres affections (maladie auto-immune, infection, grossesse, médicaments, cancer, greffe d'organe). Les facteurs déclenchant les poussées sont multiples (infection, grossesse, certains médicaments comme les thiénopyridines) et restent souvent non identifiés. Le diagnostic du PTT est parfois difficile à établir en raison de l'absence de spécificité de ses signes clinico-biologiques et ses diagnostics différentiels sont donc nombreux. En l'absence de traitement urgent et adapté dont le prérequis est bien entendu un diagnostic précis, les poussées de PTT ont un pronostic très sévère conduisant au décès dans $90 \%$ des cas. Depuis 1990, l'utilisation empirique de la plasmathérapie (idéalement échanges plasmatiques) a révolutionné le pronostic des poussées de PTT en permettant un taux de survie de $90 \%[1,30]$.

Depuis sa première description clinique en 1924 [31], la physiopathologie du PTT est restée totalement obscure jusqu'en 1982, date à laquelle il a été rapporté que les thrombus plaquettaires présents de manière diffuse dans les microvaisseaux des patients atteints de PTT avaient la particularité d'être très riches en multimères de THPM du VWF [32]. Ce n'est que 15 ans plus tard, en 1997, lorsqu'ADAMTS13, la métalloprotéase responsable de la régulation de la taille des multimères du VWF, a été découverte, que la physiopathologie du PTT a pu être élucidée [30]. En effet, chez les patients atteints de PTT, il existe dans $90 \%$ des cas un déficit fonctionnel sévère (activité inférieure à $10 \%$ ) d'ADAMTS13 qui entraîne l'accumulation anormale dans le plasma de multimères de THPM du VWF. Ce VWF «hyperadhésif » est responsable de la formation spontanée de thrombus plaquettaires qui vont obstruer la microcirculation sanguine et provoquer la poussée de PTT. Le déficit sévère en ADAMTS13 est le plus souvent acquis par le biais d'auto-Ac antiADAMTS13 (PTT acquis auto-immun représentant plus de 9 cas de PTT déficitaires en ADAMTS13 sur 10, initialement décrit sous le nom de syndrome de Moschovitz [31]) mais il peut être aussi héréditaire de transmission autosomique récessive (moins de $10 \%$ des cas de PTT déficitaire en ADAMTS13) par le biais de mutations bialléliques du gène d'ADAMTS13 (maladie orpheline appelée syndrome d'Upshaw-Schulman) [5].

\section{ADAMTS13, cible de l'auto-immunité : le modèle du PTT acquis idiopathique}

Plus de $90 \%$ des cas de PTT liés à un déficit fonctionnel sévère en ADAMTS13 correspondent à une maladie auto-immune à part entière due à l'apparition pathologique d'auto-Ac spécifiquement dirigés contre ADAMTS13 [33], qu'il s'agisse d'une forme idiopathique de PTT ou d'un PTT survenant dans un contexte clinique (notamment autre maladie autoimmune) préexis- 
tant. Ces auto-Ac anti-ADAMTS13 sont polyclonaux, reconnaissent constamment un ou plusieurs épitopes du domaine riche en cystéines d'ADAMTS13 [33]. Ils sont de type IgG dans près de $90 \%$ des cas, beaucoup plus rarement de type IgM ou IgA mais alors toujours associés à la présence d'une IgG [34]. Au plan fonctionnel, deux types d'auto-Ac anti-ADAMTS13 ont été décrits $[33,34]$ : des Ac neutralisants (ou inhibiteurs) qui agissent par inhibition directe du site catalytique d'ADAMTS13 et des Ac non neutralisants qui agissent en se complexant à ADAMTS13 et accélèrent ainsi sa clairance. Ces deux mécanismes coexistent très souvent chez les patients atteints de PTT acquis. Les auto-Ac anti-ADAMTS13 peuvent donc être mesurés soit par des méthodes immunologiques (ELISA mettant en jeu de I'ADAMTS13 recombinante de type sauvage et une révélation par des Ac anti-IgG humaine), soit par des tests fonctionnels (mesure de l'activité résiduelle d'ADAMTS13 d'un mélange de plasma témoin et de plasma testé préchauffé à $56^{\circ} \mathrm{C}$ afin de dissocier d'éventuels complexes immuns) $[34,35]$. Globalement, une IgG inhibitrice anti-ADAMTS13 est retrouvée dans près de $80 \%$ des PTT acquis avec déficit fonctionnel sévère en ADAMTS13 lors des poussées [34, 35].

La pertinence clinique de ces autoAc anti-ADAMTS13 est clairement établie : ils sont responsables du déficit fonctionnel sévère en ADAMTS13 observé lors des poussées de PTT acquis [33-35] et ils disparaissent le plus souvent en rémission clinique après traitement (plasmathérapie éventuellement associée à un traitement immmunomodulateur) parallèlement à une normalisation de l'activité d'ADAMTS13 [34, 35]. Chez certains patients cependant, malgré la rémission clinique, ces auto-Ac persistent, et cela parallèlement à une activité d'ADAMTS13 qui reste indétectable $[30,34,35]$. Un certain nombre de questions restent néanmoins en suspens quant à la valeur pronostique de ces auto-Ac anti-ADAMTS13 dans le PTT acquis lié à un déficit fonctionnel sévère en ADAMTS13 : leurs caractéristiques (titre d'IgG, caractère inhibiteur ) corrèlent-elles avec l'intensité du tableau clinique inaugural, la réponse thérapeutique de la poussée ou encore la fréquence des rechutes ultérieures? Plusieurs études prospectives ciblées sur les cohortes nationales de patients atteints de PTT lié à un déficit fonctionnel sévère en ADAMTS13 sont en cours afin de tenter de répondre à ces questions cruciales qui permettront peut-être d'utiliser les auto-Ac anti-ADAMTS13 comme marqueur pronostique et indicateur de choix thérapeutique à court et moyen termes. Audelà du modèle de pathologie humaine, l'imputabilité du mécanisme Ac-dépendant dans le développement du PTT acquis a été récemment renforcée grâce à un modèle animal (babouin) chez lequel l'injection d'Ac monoclonaux murins anti-ADAMTS13 est capable d'induire un PTT [36]. Une autre question en suspens concerne les mécanismes à l'origine de la rupture de la tolérance du système immunitaire vis-à-vis d'ADAMTS13 : il semble exister des gènes de susceptibilité, notamment dans le système HLA [37] ; la présence de certaines mutations hétérozygotes d'ADAMTS13 est une autre piste prometteuse [38]. Enfin, de manière très intéressante, chez certains patients atteints de PTT acquis idiopathique, il semble que les auto-Ac anti-ADAMTS13 soient associés d'emblée ou précèdent de quelques mois (années) l'apparition d'autres auto-Ac notamment de facteurs anti-nucléaires [39].

\section{ADAMTS13, cible de mutations génétiques: le modèle du PTT héréditaire ou syndrome d'Upshaw-Schulman}

Moins de $10 \%$ des cas de PTT liés à un déficit fonctionnel sévère en ADAMTS13 sont dus à des mutations bialléliques du gène codant ADAMTS13 (forme héréditaire du PTT appelée syndrome d'Upshaw-Schulman [USS]) [5]. Une centaine de mutations différentes d'ADAMTS13 sont recensées à ce jour dans la littérature internationale chez les patients atteints d'USS [40]. Alors que l'USS a une répartition mondiale ubiquitaire, il y a peu de mutations communes d'ADAMTS13 entre les différents pays du monde, certaines mutations étant intimement liées à certaines zones géographiques $[30,40]$. Les mutations d'ADAMTS13 sont héritées selon une transmission autosomique récessive, les patients étant le plus souvent hétérozygotes composites ou plus rarement homozygotes (en particulier lorsqu'il existe une consanguinité familiale). Ces mutations sont réparties sur toute la longueur du gène sans hotspot (Figure 4). II s'agit de mutations faux-sens (substitutions) dans $70 \%$ des cas et de mutations de terminaison (codons stop, anomalies du site d'épissage, insertions, délétions partielles) dans $30 \%$ des cas. Toutes les combinaisons possibles de mutations sont retrouvées chez les patients USS mais une délétion totale d'ADAMTS13 n'a jamais été décrite, ce qui suggère que celle-ci est probablement létale $[5,30,40]$. Dans la grande majorité des cas, le mécanisme du déficit fonctionnel sévère en ADAMTS13 induit par les mutations de son gène relève d'un déficit quantitatif lié à un défaut de synthèse et/ou de sécrétion de la protéine [30]. Étayer les relations phénotype/génotype dans le USS demeure très difficile en raison du faible nombre de patients, de la multiplicité des mutations et de l'hétérogénéité de l'expression clinique (âge de révélation de la maladie, sévérité et fréquence des poussées), cela même au sein d'une famille donnée [30, 40]. Néanmoins, alors que la première poussée d'USS a lieu le plus souvent dans la petite enfance (souvent dès la naissance), de très rares formes se révèlent à l'âge adulte et semblent associées à une mutation particulière (R1060W) [38]. Parmi les autres facteurs susceptibles de moduler l'expression des mutations d'ADAMTS13 dans I'USS, citons certains polymorphismes d'ADAMTS13 [41] et aussi des gènes impliqués dans la régulation du taux de VWF plasmatique [42]. Cette implication du taux de VWF dans la susceptibilité au PTT sur un fond de déficit sévère en ADAMTS13 est clairement mise en évidence dans les modèles animaux de souris invalidées pour le gène d'ADAMTS13 où l'intensité de l'expression clinique du PTा est intimement corrélée au taux de VWF plasmatique circulant [43]. 


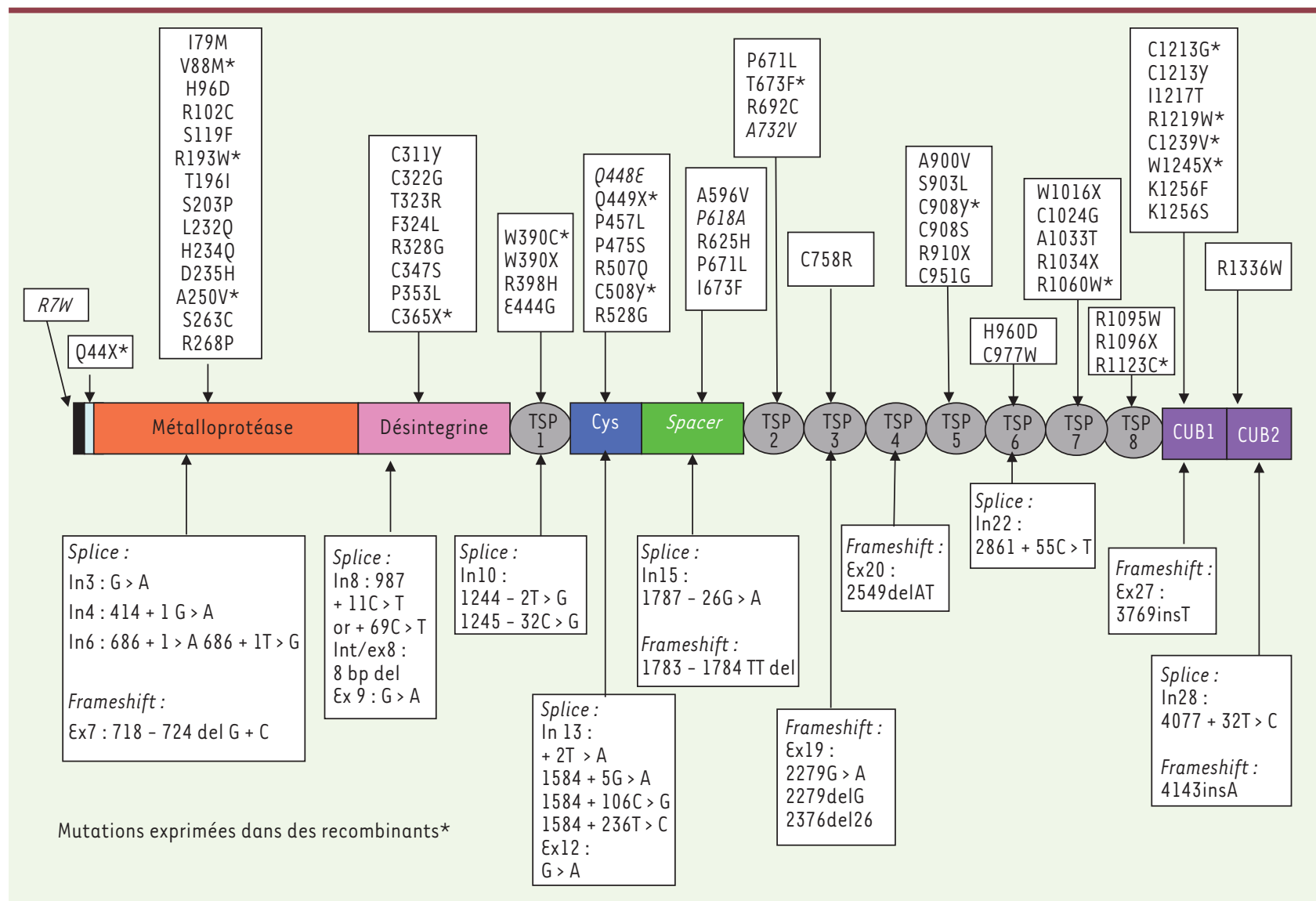

Figure 4. Mutations du gène d'ADAMTS13 dans le syndrome d'Upshaw-Schulman (forme héréditaire du PTT). Une centaine de mutations du gène d'ADAMTS13 ont été décrites dans le syndrome d'Upshaw-Schulman. Ces mutations sont localisées sur toute la longueur du gène (absence de hotspot) et conduisent principalement à des substitutions d'aa ou à des codons stop (indiqués sur la partie supérieure de la figure). عlles consistent plus rarement en des délétions, insertions ou des mutations de sites d'épissage (indiquées sur la partie inférieure de la figure).

\section{ADAMTS13, un outil thérapeutique?}

L'efficacité de la plasmathérapie utilisée initialement de manière empirique depuis 1990 dans le PTT est aujourd'hui clairement imputable à la présence d'ADAMTS13 active dans les produits plasmatiques utilisés dans cette indication $[1,30]$. Depuis quelques années, la recherche pharmaceutique travaille au développement d'une ADAMTS13 purifiée à partir de plasma humain et d'une ADAMTS13 recombinante : ces produits ne sont pas encore disponibles mais quelques études préliminaires réalisées in vitro montrent des résultats prometteurs [44]. Une autre approche qui repose sur la thérapie génique a également été initiée chez l'animal permettant une restauration de taux sanguins d'ADAMTS13 détectables chez des souris invalidées pour le gène codant ADAMTS13 [45-47].

\section{Conclusion}

Depuis les années 2000, la découverte d'ADAMTS13 a essentiellement permis une avancée médicale cruciale dans la compréhension de la physiopathologie, le diagnostic et le traitement d'une maladie rare, le PTT [48]. Plus récemment, le rôle d'ADAMTS13 dans la thrombose artérielle a été évoqué selon un concept qui souligne sa position de lien entre l'inflammation et la thrombose [49]. Au-delà du modèle « vertical » du PTT, cette dernière observation ouvre donc de nouveaux champs d'investigation pour la recherche dédiée à ADAMTS13, plus particulièrement ciblés sur les pathologies vasculaires [50].

\section{SUMMARY}

\section{ADAMTS13, von Willebrand}

factor specific cleaving protease

ADAMTS13 (A disintegrin and metalloprotease with thrombospondin type 1 repeats) is the specific von Willebrand factor (VWF)-cleaving protease. ADAMTS13 was partially purified from human plasma in 1996 and its gene was cloned in 2001. In case of vascular injury, multimeric VWF is the mediator of both platelet adhesion to 
the sub-endothelium and platelet aggregation within the microvessels at high shear rates of blood flow. ADAMTS13 regulates VWF adhesive capacity by reducing the size of VWF multimers. A severe functional deficiency of ADAMTS13 (activity lower than 10\%) is associated with most cases of thrombotic thrombocytopenic purpura (TTP), a thrombotic microangiopathy characterized by the spontaneous formation, within the microcirculation, of VWF-rich platelet thrombi responsible for a mechanical hemolytic anemia, a consumption thrombocytopenia and a multivisceral ishemia. TTP is a rare disease (4 cases/106/ year) with a life-threatening prognosis in the absence of an appropriate treatment in emergency (plasmatherapy). In $90 \%$ of cases, TTP is acquired and related to the development of auto-antibodies to ADAMTS13. In the other cases, TTP is inherited via bi-allelic autosomic recessive mutations of ADAMTS13 gene (Upshaw-Schulman syndrome). A better characterization of ADAMTS13 structure/function combined to clinical trials led in TTP patients is crucial to evaluate the relevance of either a plasma-purified or a recombinant ADAMTS13 as a therapeutic agent. $\diamond$

\section{CONFLIT D'INTÉRÊTS}

Les auteurs déclarent n'avoir aucun conflit d'intérêts concernant les données publiées dans cet article.

\section{RÉFÉRENCES}

1. Sadler JE. Von Willebrand factor, ADAMTS13 and thrombotic thrombocytopenic purpura. Blood $2008 ; 112: 11-8$.

2. Fujikawa K, Suzuki H, McMullen B, Chung D. Purification of human von Willebrand factor-cleaving protease and its identification as a new member of the metalloprotease family. Blood 2001 ; 98 : 1662-6.

3. Zheng X, Chung D, Takayama TK, et al. Structure of von Willebrand factor-cleaving protease (ADAMTS13), a metalloprotease involved in thrombotic thrombocytopenic purpura. J Biol Chem $2001 ; 276: 41059-63$.

4. Soejima K, Mimura N, Hirashima M, et al. A novel metalloprotease synthesized in the liver and secreted into the blood: possibly, the von Willebrand factor-cleaving protease? J Biochem 2001 ; $130: 475-80$.

5. Levy GG, Nichols WC, Lian $E C$, et al. Mutations in a member of the ADAMTS gene family cause thrombotic thrombocytopenic purpura. Nature $2001 ; 413: 488-94$.

6. Zhou W, Inada M, Lee TP, et al. ADAMTS13 is expressed in hepatic stellate cells. Lab Invest 2005; $85: 780-8$.

7. Turner N, Nolasco L, Tao Z, et al. Human endothelial cells synthesize and release ADAMTS13. J Thromb Haemost 2006 ; 4 : 1396-404.

8. Liu L, Choi H, Bernardo A, et al. Platelet-derived VWF-cleaving metalloprotease ADAMTS-13. J Thromb Haemost $2005 ; 3: 2536-44$.

9. Furlan M, Robles R, Lämmle B. Partial purification and characterization of a protease from human plasma cleaving von Willebrand factor to fragments produced by in vivo proteolysis. Blood 1996 ; $87: 4223-34$.

10. Tsaï HM. Physiologic cleavage of von Willebrand factor by a plasma protease is dependent on its conformation and requires calcium ion. Blood $1996 ; 87: 4235-44$

11. Furlan M, Robles R, Morselli B, et al. Recovery and half-life of von Willebrand factor-cleaving protease after plasma therapy in patients with thrombotic thrombocytopenic purpura. Thromb Haemost $1999 ; 81: 8-13$.

12. Jenkins PV, Pasi KJ, Perkins SJ. Molecular modeling of ligand and mutation sites of the type A domains of human von Willebrand factor and their relevance to von Willebrand's disease. Blood 1998 ; 91 : 2032-44.

13. Majerus $E M$, Anderson PJ, Sadler JE. Binding of ADAMTS13 to von Willebrand factor. J Biol Chem $2005 ; 280: 71773-8$.

14. Padilla A, Moake JL, Bernardo A, et al. P-selectin anchors newly released ultralarge von Willebrand factor multimers to the endothelia cell surface. Blood $2004 ; 103: 2150-6$.

15. Nishio K, Anderson PJ, Zheng XL, Sadler JE. Binding of platelet glycoprotein Ib $\alpha$ to von Willebrand factor domain Al stimulates the cleavage of the adjacent domain A2 by ADAMTS13. Proc Natl Acad Sci USA $2004 ; 101: 10578-83$.
16. Majerus EM, Zheng XL, Tuley EA, Sadler JE. Cleavage of the ADAMTS13 propeptide is not required for protease activity. J Biol Chem $2003 ; 278$ : 46643-8.

17. Soejima K, Matsumoto M, Kokame K, et al. ADAMTS-13 cysteine-rich/spacer domains are functionally essential for von Willebrand factor cleavage. Blood $2003 ; 102: 3232-7$.

18. Zhang P, Pan W, Rux AH, et al. The cooperative activity between the carboxyterminal TSPI repeats and the CUB domains of ADAMTS13 is crucial for recognition of von Willebrand factor under flow. Blood 2007 ; 110 : 1887-94.

19. Davis AK, Makar RS, Stowell CP, et al. ADAMTS13 binds to CD36: a potential mechanism for platelet and endothelial localization of ADAMTS13. Transfusion $2009 ; 49: 206-13$.

20. Zanardelli $S$, Chion AC, Groot $\varepsilon$, et al. A novel binding site for ADAMTS13 constitutively exposed on the surface of globular VWF. Blood $2009 ; 114$ : 2819-28.

21. Tao Z, Wang $\mathrm{Y}$, Choi $\mathrm{H}$, et al. Cleavage of ultralarge multimers of von Willebrand factor by $\mathrm{C}$-terminal-truncated mutants of ADAMTS- 13 under flow. Blood $2005 ; 106$ : 141-3.

22. Gao W, Anderson PJ, Majerus EM, et al. Exosite interactions contribute to tension-induced cleavage of von Willebrand factor by the antithrombic ADAMTS13 metalloprotease. Proc Natl Acad Sci USA 2006 ; 103 : 19099 104

23. Veyradier A, Girma JP. Assays of von ADAMTS13 activity. Semin Hematol $2004 ; 41: 41-7$.

24. Kokame K, Nobe $Y$, Kokubo $Y$, et al. FRETS-VWF73, a first fluorogenic substrate for ADAMTS13 assay. BrJ Haematol 2005 ; 129 : 93-100.

25. Tripodi A, Chantarangkul V, Böhm M, et al. Measurement of von Willebrand factor-cleaving protease (ADAMTS-13): results of an international collaborative study involving 11 methods testing the same set of coded plasmas. J Thromb Haemost 2004 ; 2 : 1601-9.

26. Kremer-Hovinga J, Mottini M, Lämmle B. Measurement of ADAMTS13 activity in plasma by the FRETS-VWF73 assay: comparison with other assay methods. J Thromb Haemost $2006 ; 4: 1146-8$.

27. Mannucci PM, Canciani MT, Forza I, et al. Changes in health and disease of the metalloprotease that cleaves von Willebrand factor. Blood $2001 ; 98$ : 2730-5.

28. Schmugge M, Dunn MS, Amankwah KS, et al. The activity of the von Willebrand factor-cleaving protease ADAMTS-13 in newborn infants. J Thromb Haemost $2003 ; 2: 228-33$.

29. Sanchez-Luceros A, Farias CE, Amaral MM, et al. Von Willebrand factorcleaving protease (ADAMTS13) activity in normal non-pregnant women, pregnant and post-delivery women. Thromb Haemost 2004 ; 92 : 1320-6.

30. Zheng XL, Sadler JE. Pathogenesis of thrombotic microangiopathies. Annu Rev Pathol 2008 ; $3: 249-77$.

31. Moschcovitz $\varepsilon$. Hyaline thrombosis of the terminal arterioles and capillaries: a hitherto undescribed disease. Proc Ny Pathol Soc $1924 ; 24: 21-4$.

32. Moake JL, Rudy CK, Troll JH, et al. Unusually large plasma factor VIII: von Willebrand factor multimers in chronic relapsing thrombotic thrombocytopenic purpura. N Engl J Med 1982 ; 307 : 1432-5.

33. Rieger M, Mannucci PM, Kremer Hovinga JA, et al. ADAMTS13 antibodies in patients with thrombotic microangiopathies and other immunomediated diseases. Blood $2005 ; 106: 1262-7$.

34. Ferrari S, Scheiflinger F, Rieger M, et al. Prognostic value of anti-ADAMTS13 antibodies feature (Ig isotype titer and inhibitory effect) in a cohort of 35 adult French patients undergoing a first episode of thrombotic microangiopathy with an undetectable ADAMTS13 activity. Blood 2007 ; $109:$ 2815-22.

35. Scully M. Inhibitory anti-ADAMTS13 antibodies: measurement and clinical application. Blood Rev $2010 ; 24$ : 11-6.

36. Feys HB, Roodt J, Vandeputte N, et al. Thrombotic thrombocytopenic purpura directly linked with ADAMTS13 inhibition in the baboon (Papio ursinus). Blood 2010 ; 116 : 2005-10.

37. Coppo P, Busson M, Veyradier A, et al. HLA-DRB1*11: a strong risk factor for acquired severe ADAMTS13 deficiency-related idiopathic thrombotic thrombocytopenic purpura in Caucasians. J Thromb Haemost 2010; 8 : 856-9.

38. Camilleri RS, Cohen H, Mackie IJ, et al. Prevalence of the ADAMTS13 missense mutation R1060W in late onset adult thrombotic thrombocytopenic purpura. J Thromb Haemost 2008 ; 6 : 331-8.

39. Coppo P, Benghoufa D, Veyradier A, et al. Prevalence and clinical significance of anti-nuclear antibodies in adult thrombotic microangiopathies. Medicine (Balt) $2004 ; 83: 233-44$. 
40. Lotta LA, Garagiola I, Palla R, et al. ADAMTS13 mutations and polymorphisms in congenital thrombotic thrombocytopenic purpura. Hum Mutat $2010 ; 31: 11-9$.

41. Plaimauer B, Fuhrmann J, Mohr G, et al. Modulation of ADAMTS13 secretion and specific activity by a combination of common amino acid polymorphisms and a missense mutation. Blood 2006 ; $107: 118-25$.

42. Westrick RJ, Ginsburg D. Modifier genes for disorders of thrombosis and hemostasis. J Thromb Haemost $2009 ; 7$ (suppl 1):132-5.

43. Chauhan AK, Walsh MT, Zhu G, et al. The combined roles of ADAMTS13 and VWF in murine models of TTP, endotoxemia, and thrombosis. Blood 2008; $111: 3452-7$

44. Plaimauer B, Kremer Hovinga J, Juno C, et al. Recombinant ADAMTS13 normalizes von Willebrand factor-cleaving activity in plasma of acquired TTP patients by overriding inhibitory antibodies.J Thromb Haemost $2011 ; 9$ : 936-44.

45. Laje P, Shang D, Cao W, et al. Correction of murine ADAMTS13 deficiency by hematopoietic progenitor cell-mediated gene therapy. Blood 2009; $113: 2172-80$.

46. Niiya M, Endo M, Shang D, et al. Correction of ADAMTS13 deficiency by in utero gene transfer of lentiviral vector encoding ADAMTS13 genes. Mol Ther $2009 ; 17: 34-41$.
47. Trionfini P, Tomasoni S, Galbusera M, et al. Adenoviral-mediated gene transfer restores plasma ADAMTS13 antigen and activity in ADAMTS13 knockout mice. Gene Ther 2009 ; 16 : 1373-9.

48 Girma JP, Veyradier A, Meyer D. ADAMTS 13, la protéase spécifique du facteur Willebrand. Med Sci (Paris) $2002 ; 18: 15-7$.

49. Chauhan AK, Kisucka J, Brill A, et al. ADAMTS13: a new link between thrombosis and inflammation.J Exp Med 2008 ; $205: 2065-74$

50. Fujioka M, Hayakawa K, Mishima K, et al. ADAMTS13 gene deletion aggravates ischemic brain damage: a possible neuroprotective role of ADAMTS13 by ameliorating postischemic hypoperfusion. Blood $2010 ; 115$ $1650-3$.

TIRÉS À PART

A. Veyradier

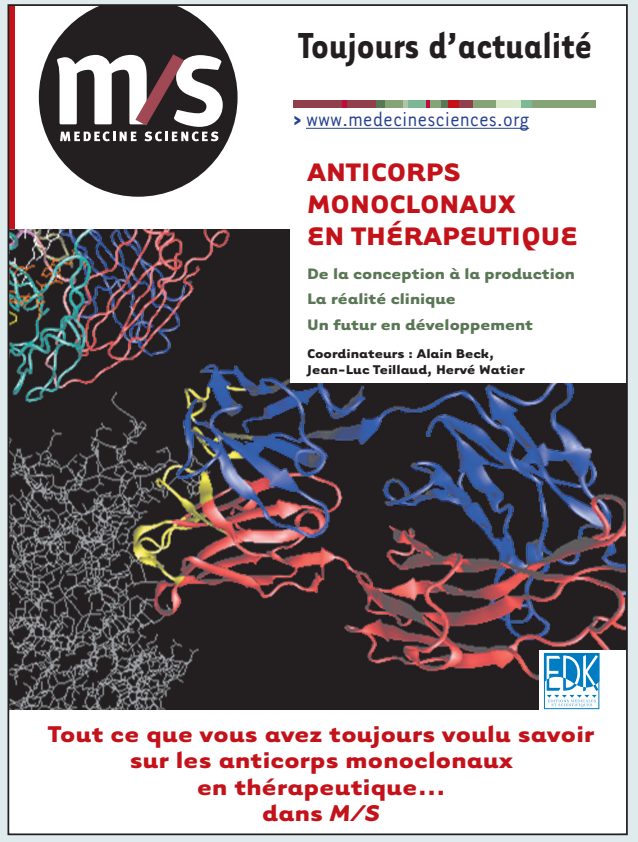

Tout ce que vous avez toujours voulu savoir sur les anticorps monoclonaux en thérapeutique... dans Médecine/Sciences. Pourquoi un numéro spécial de Médecine/Sciences sur les anticorps monoclonaux thérapeutiques? II nous a semblé que le moment était venu de dresser un état des lieux de ces biomédicaments qui prennent désormais une place considérable - et croissante dans les traitements de maladies souvent lourdes et désespérantes. Ce voyage que nous vous proposons à la découverte du monde des anticorps thérapeutiques nous a appris, ou plutôt rappelé, une évidence : les compétences en France sont fortes et nombreuses, qu'elles soient académiques ou industrielles, biotechnologiques ou cliniques. Le paysage français, trop longtemps discret, bruisse désormais de mille initiatives balayant de multiples aspects des anticorps thérapeutiques : études précliniques et cliniques menées avec de nouveaux anticorps dirigés contre des cibles originales, développement de nouveaux formats d'anticorps ou d'anticorps optimisés reposant sur des études structurales et fonctionnelles sophistiquées, recherche active de cibles pertinentes, mise au point de méthodologies de bioproduction, de couplage, etc. L'expansion industrielle rapide de ce champ est un défi que peut et doit relever notre pays, défi tant scientifique qu'économique, avec ses combats pour la propriété intellectuelle et pour l'emploi de nos jeunes scientifiques.

Alain Beck, Jean-Luc Teillaud, Hervé Watier

À retourner à EDK, 25, rue Daviel - 75013 Paris, France

Tél. : 0158101905 - Fax : 0143293262 - E-mail : edk@edk.fr

NOM :

Prénom :

Adresse :

Code postal :

Ville :

Pays :

Fonction :

Je souhaite recevoir $\mathbf{M} / \mathbf{S} \mathbf{n}^{\circ} \mathbf{1 2}$ - décembre 2009 (Anticorps monoclonaux en thérapeutique) : $25 €+3 €$ de port $=\mathbf{2 8} €$ TTC en exemplaire, soit un total de $€$

Par chèque, à l'ordre de $\mathbf{E} \mathbf{D} \mathbf{K}$

Par carte bancaire : $\square$ Visa Eurocard/Mastercard Carte $n^{\circ} 11_{1}|1| \begin{array}{lllllllllllllll}1 & 1 & 1 & 1 & 1 & 1 & 1 & 1 & 1 & 1 & 1 & 1 & 1 & 1\end{array}$

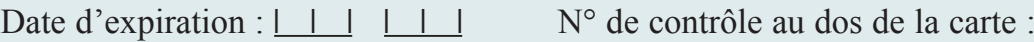

Signature : 